\title{
Quantification of UV-Visible and Laser Spectroscopic Techniques for Materials Accountability and Process Control
}

\section{Fuel Cycle R\&D}

Dr. Ken Czerwinski University of Nevada, Las Vegas

In collaboration with:

Argonne National Laboratory Oak Ridge National Laboratory Pacific Northwest National Laboratory

Daniel Vega, Federal POC Michael Miller, Technical POC

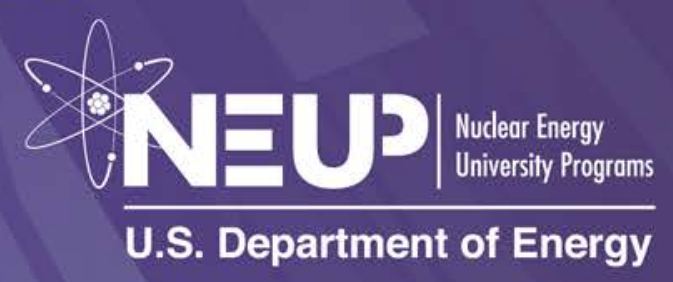


Quantification of UV-Visible and Laser Spectroscopic Techniques for Materials

Accountability and Process Control (09-350)

Quarterly and Final Report (July 2012 to Sept 2012)

Ken Czerwinski, Phil Weck, University of Nevada, Las Vegas

Graduate Students: William Kerlin

Collaborators: Samuel Bryan, PNNL, Robert Jubin, ORNL, Monica Regalbuto, ANL

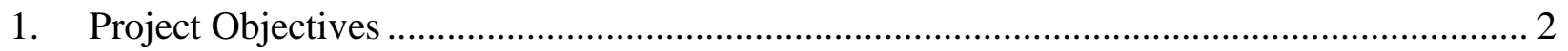

2. Path to Accomplish Scope ........................................................................................ 3

2.1. Task 1: UV-Visible spectroscopy of U and Pu under UREX, TALSPEAK, and TRUEX

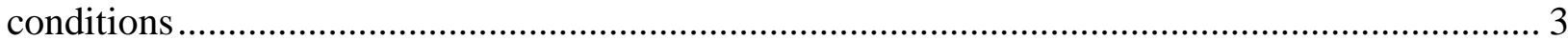

2.2. Task 2: U and Cm TRLFS studies under UREX+ conditions ....................................... 3

2.3. Task 3: Application of project data to on-line systems................................................ 4

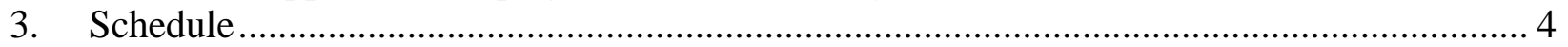

4. Work Performed in Previous Quarter (April 2012- June 2012) ........................................ 4

5. $\quad$ Work performed in Current Quarter (July 2012-September 2012) .................................... 5

6

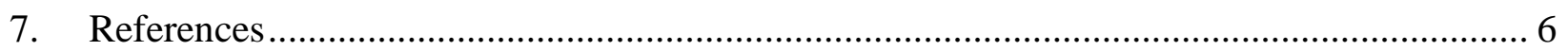




\section{Project Objectives}

Ultraviolet-Visible Spectroscopy (UV-Visible) and Time Resolved Laser Fluorescence Spectroscopy (TRLFS) optical techniques can permit on-line, real-time analysis of the actinide elements in a solvent extraction process. UV-Visible and TRLFS techniques have been used for measuring the speciation and concentration of the actinides under laboratory conditions. These methods are easily adaptable to multiple sampling geometries, such as dip probes, fiber-optic sample cells, and flow-through cell geometries. To fully exploit these techniques for GNEP applications, the fundamental speciation of the target actinides and the resulting influence on spectroscopic properties must be determined. Through this effort detection limits, process conditions, and speciation of key actinide components can be establish and utilized in a range of areas of interest to GNEP, especially in areas related to materials accountability and process control.

In this project UV-Visible and TRLFS will be evaluated for use in proposed GNEP solvent extraction based separations (Figure 1). Research in the PI's radiochemistry laboratory is currently examining the efficacy of UV-Visible spectroscopy to evaluate U and plutonium speciation under UREX conditions. Studies are also ongoing using TRLFS to evaluate Cm speciation and concentration in the TALSPEAK process. The success of the uranyl and plutonium nitrate UV-Visible spectroscopy study supports the utility and continued exploration of spectroscopic methods for the evaluation of actinide concentrations and solution conditions for other aspects of the UREX+ solvent extraction scheme. Studies in this project will extend the current project (Project 08-6046, funded for 1 year) to examine $U$ and Pu absorbance in the TRUEX and TALSPEAK, detailed examination of Cm in TRUEX and TALSPEAK, $\mathrm{U}$ laser fluorescence, and application of project data to contactors. Peak ratios will be determined as a function of solution concentrations for the UV-Visible spectroscopy studies. The use of TRLFS to examine $\mathrm{Cm}$ and $\mathrm{U}$ will provide data to evaluate lifetime, peak location, and peak ratios (mainly for $\mathrm{U}$ ). The bases for the spectroscopic techniques have been investigated in the UNLV laboratory, providing fundamental evidence for the utility of the application. Through this research effort, tools and spectroscopic techniques will be developed to evaluate and determine solution extraction conditions and concentrations of $\mathrm{U}, \mathrm{Pu}$ and $\mathrm{Cm}$ in extraction processes, addressing areas on process control and materials accountability.

The university PI's program is actively engaged with DOE scientists on spectroscopic studies of extraction systems including UV-Visible spectroscopy of $U$ and Tc separations the university UNLV providing support for both student interactions at the DOE facilities and DOE researchers at university laboratories. It is expected that the developed spectroscopic techniques will be examined in detail, providing a foundational chemical approach based speciation to interpret the spectra. Through the collaborators the application of the developed techniques to DOE facilities, such as CETE, will be accomplished. This project will also train the next generation of radiochemistry researchers necessary for the long-term success of GNEP and other DOE projects. The research team will include graduate students in the university radiochemistry Ph.D. program. This project will expose the students to DOE researchers and facilities, providing a basis for introducing future researchers to DOE needs and scientific opportunities.

The project successfully determined a route to evaluate uranium, nitrate, and acid concentration from absorbance spectroscopy. The method is described in thesis works ${ }^{1,2,3}$, publications ${ }^{4}$, and previous quarterly reports. The studies demonstrated how uranium absorbance peak ratios provide sufficient information to determine speciation and chemical composition of aqueous solution on-line and in real time. The use of density functional theory 
shows how uranium speciation impacted spectroscopic behavior. The fluorescence properties of lanthanides and actinides were shown to provide extremely sensitive methods for determining concentrations of these metal ions in a number of different extraction conditions.

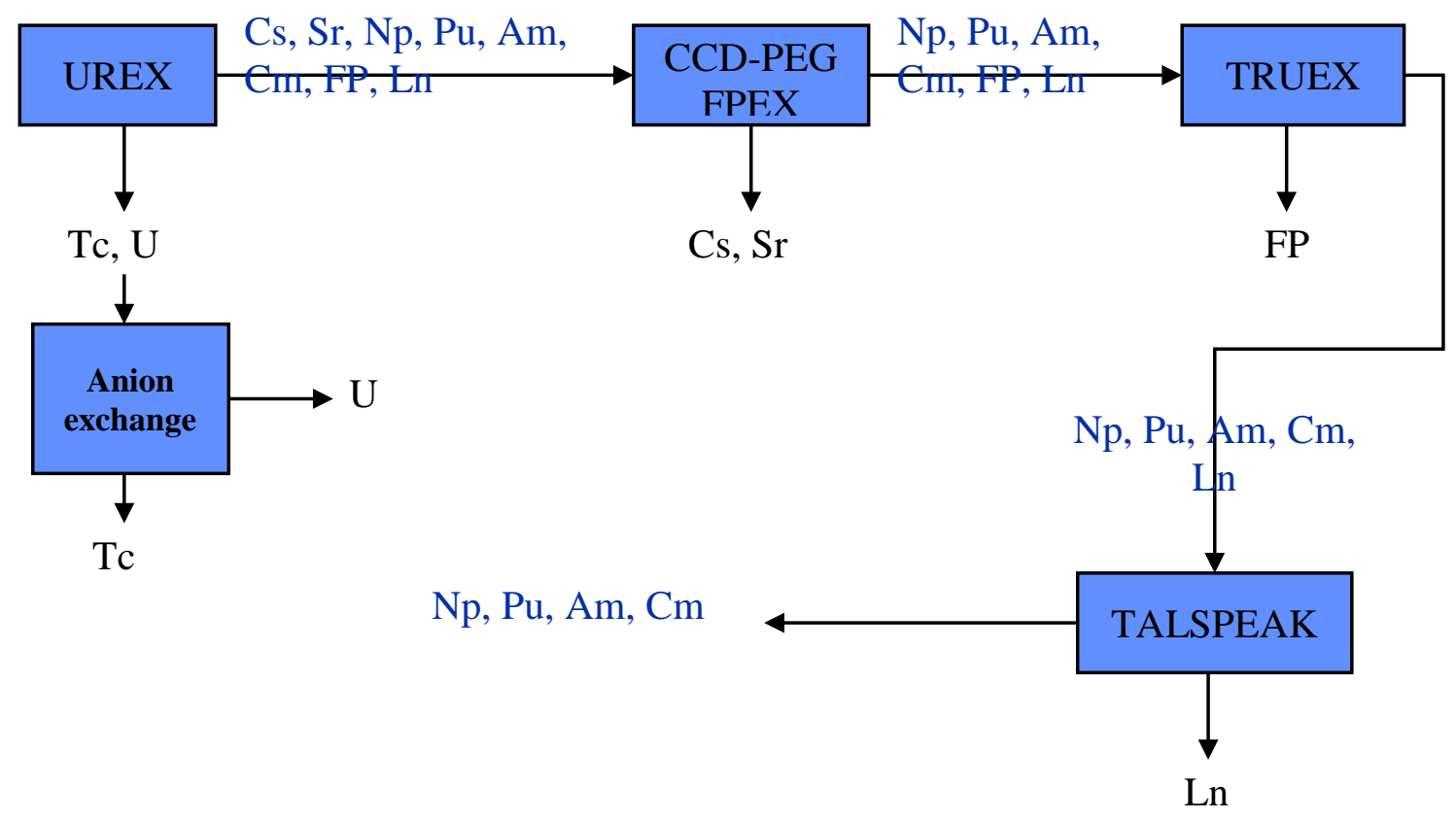

Figure 1. Schematic of potential UREX based separation

\section{Path to Accomplish Scope}

\subsection{Task 1:_UV-Visible spectroscopy of U and Pu under UREX, TALSPEAK, and TRUEX conditions}

The UV-Visible absorbance spectroscopy of $\mathrm{U}$ and $\mathrm{Pu}$ will be examined under this task and based upon ongoing experiments. Peak ratios will be determined as a function of solution conditions to assess actinide speciation, actinide concentration, and ligand concentrations. The studies will also include any impact Pu has on the uranyl system as well as the impact of uranyl on Pu spectroscopy. The expected molar absorptivities for $\mathrm{Pu}(\mathrm{IV})$ are around $55 \mathrm{~L} \mathrm{~mol}^{-1} \mathrm{~cm}^{-1}$ at $470 \mathrm{~nm}$ and $35 \mathrm{~L} \mathrm{~mol}^{-1} \mathrm{~cm}^{-1}$ at $650 \mathrm{~nm}$, requiring concentrations in the $\mathrm{mM}$ range or the use of a waveguide. Both techniques will be utilized. The PI's radiochemistry program has already such experiments with $\mathrm{Pu}$ and $\mathrm{U}$.

\subsection{Task 2: U and Cm TRLFS studies under UREX+ conditions}

The use of TRLFS to examine $\mathrm{U}$ and Cm concentration and speciation under extraction conditions applicable to TRUEX and TALSPEAK will be investigated in this task, expanding on the $\mathrm{Tb}$ and $\mathrm{Cm}$ TALSPEAK studies currently under investigation. The basis for this technique has already been demonstrated in the PI's laboratory with this task an expansion of an initial 1 year GNEP project. The U and Cm spectroscopy will be examined in both the aqueous and organic phase. Previous studies have demonstrated limitations in organic phase U fluorescence due to quenching and redox reactions. A range of nitric acid aqueous phases, based on the 
output from CCD-PEG, FPEX, and NPEX, will be assessed. The organic phase will be based on the TRUEX and TALSPEAK schemes. Curium excitation will use both $434 \mathrm{~nm}$ and $375 \mathrm{~nm}$, with fluorescence monitored near $595 \mathrm{~nm}$. Uranium excitation will occur near $425 \mathrm{~nm}$. As only a single fluorescence peak for $\mathrm{Cm}$ is expected for a given species, peak ratios will not be determined unless multiple Cm species are present. Efforts in this task will focus on measuring the exact fluorescence wavelength, intensity, and lifetime against solution conditions. The U and Cm concentration and solution condition can be determined by the task data.

\subsection{Task 3: Application of project data to on-line systems}

Recent collaborative efforts by the project members have involved utilizing spectroscopic techniques in on-line systems. This effort will be extended to the use of flow cells and contactors at DOE facilities. In summer 2009 graduate students involve in on-going research on uranium spectroscopy performed studies at a third DOE laboratory using their contactors. These studies will extend previous efforts and focus on overcoming plug flow and other engineering issues. The task effort will evaluate the efficacy of flow through cells coupled to slip streams in addition to dip probe configurations.

\section{Schedule}

- $\quad$ Task 1: UV-Visible spectroscopy of U and Pu under UREX, TALSPEAK, and TRUEX conditions

Months 0-12: Finalization of $U$ and Pu studies under UREX conditions

Months 0-24: U and Pu studies under TALSPEAK conditions

Months 6-36: $\mathrm{U}$ and Pu studies under TRUEX conditions

- $\quad$ Task 2: U and Cm TRLFS studies under UREX+ conditions

Months 0-18: Evaluation on U fluorescence spectroscopy for UREX conditions

Months 0-15: Completion of Cm TALSPEAK spectroscopic studies

Months 6-24: Evaluation on U fluorescence spectroscopy for TALSPEAK conditions

Months 12-36: Evaluation of Cm spectroscopy under TRUEX conditions

Months 12-36: Evaluation on U fluorescence spectroscopy for TRUEX conditions

- Task 3: Application of project data to on-line systems

Months 9-36: Application of project data to flow cell studies

Months 12-36: Application of project data to contactor studies performed in conjunction with DOE partners.

\section{Work Performed in Previous Quarter (April 2012- June 2012)}

Research focused on Cm laser spectroscopy. The examined systems include interactions with CMPO and TBP. These ligands are used in past and existing separation systems. These ligands are also part of mixed systems currently under exploration for group actinide separation. The results demonstrate the utility of $\mathrm{Cm}$ for online analysis of processes similar to TALSPEAK and TRUEX. Under the examined system submicromolar Cm concentrations were sufficient for laser spectroscopic analysis. This concentration is orders of magnitude lower than those anticipated in separation processes requiring on-line, real time analysis.

First-principles study has been carried out to gain fundamental understanding of complexation of lanthanide ions with 5,6-dialkyl-2,6-bis(1,2,4-triazin-3-yl)pyridine (RBTP, R=H (HBTP), Methyl (MeBTP), Ethyl (EtBTP)) derivatives studied in acetonitrile medium experimentally at the UNLV radiochemistry group by using electrospray ionization mass 
spectrometry (ESI-MS), time resolved laser induced fluorescence spectroscopy (TRLFS) and UV-Visible spectrophotometric method. The experimental efforts to unravel the effect of nitrate ion on their complexation behavior indicate clearly the formation of lower stoichiometric complexes in presence of excess nitrate ion. Different $\mathrm{Am}^{3+}$ and $\mathrm{Ln}^{3+}$ complexes were observed with RBTP ligands in presence of 0.01M tetramethyl ammonium nitrate (TMAN). Formation of higher stoichiometric complexes and higher stability constants for $\mathrm{Am}^{3+}$ as compared to $\mathrm{Ln}^{3+}$ ions indicates the selectivity of these classes of ligand. Single crystal XRD study of Eu(III) complexes shows the formation of dimeric complex with HBTP and monomeric complex with EtBTP, whereas MeBTP forms both the dimeric and monomeric complexes. Density functional theory (DFT) calculations have been performed to investigate the crystal structures of the $\mathrm{Eu}$ (III) complexes of RBTP and also predict the structures of $\mathrm{Eu}^{3+}$ and $\mathrm{Am}^{3+}$ complexes observed experimentally.

\section{Work performed in Current Quarter (July 2012-September 2012)}

Two manuscripts were prepared and submitted to journals during this quarter. One paper is titled "Fluorescence and absorbance spectroscopy of the uranyl ion in nitric acid for process monitoring applications. This work presents the observation of the spectroscopic parameters (molar absorptivities, fluorescent response) of the uranyl ion across the range of conditions expected in reprocessing chemistry. From this data, a monitor using the ratio of the absorbance of the uranyl ion at 403 and $426 \mathrm{~nm}$ has been developed. This technique can the nitrate solution concentration and can be coupled with a condition appropriate molar absorptivity to determine the uranyl concentration. This method provides a reliable technique for online, real time process monitoring of the uranyl and nitrate concentration under a wide range of conditions.

The second manuscript is titled "Trivalent Actinide and Lanthanide Complexation of 5,6Dialkyl-2,6-bis(1,2,4-triazin-3-yl)pyridine (RBTP; $\mathrm{R}=\mathrm{H}$, Me, Et) Derivatives: A Combined Experimental and First-Principles Study. In this work complexations of lanthanide ions with 5,6-dialkyl-2,6-bis(1,2,4-triazin-3-yl)pyridine [RBTP; R = H (HBTP),methyl (MeBTP), ethyl (EtBTP)] derivatives have been studied in the acetonitrile medium by electrospray ionization mass spectrometry, time-resolved laser-induced fluorescence spectroscopy, and UV-vis spectrophotometric titration. These studies were carried out in the absence and presence of a nitrate ion in order to understand the effect of the nitrate ion on their complexation behavior, particularly in the poor solvating acetonitrile medium where strong nitrate complexation of hard lanthanide ions is expected. Consistent results from all three techniques undoubtedly show the formation of lower stoichiometric complexes in the presence of excess nitrate ion. This kind of nitrate ion effect on the speciation of $\mathrm{Ln}^{3+}$ complexes of RBTP ligands has not so far been reported in the literature. Different $\mathrm{Am}^{3+}$ and $\mathrm{Ln}^{3+}$ complexes were observed with RBTP ligands in the presence of $0.01 \mathrm{M}$ tetramethylammonium nitrate, and their stability constant values are determined using UV-vis spectrophotometric titrations. The formation of higher stoichiometric complexes and higher stability constants for $\mathrm{Am}^{3+}$ compared to $\mathrm{Ln}^{3+}$ ions indicates the selectivity of these classes of ligands. A single-crystal X-ray diffraction (XRD) study of europium(III) complexes shows the formation of a dimeric complex with HBTP and a monomeric complex with EtBTP, whereas MeBTP forms both the dimeric and monomeric complexes. Density functional theory calculations confirm the findings from single-crystal XRD and also predict the structures of $\mathrm{Eu}^{3+}$ and $\mathrm{Am}^{3+}$ complexes observed experimentally. 
6. Financial

\begin{tabular}{|c|c|c|c|c|r|}
\hline \multirow{2}{*}{ Q4 - Y3 } & July 2012 & Aug 2012 & Sept 2012 & QTR TOTAL & CUM TOTAL \\
\cline { 2 - 5 } & 36,456 & 36,456 & 36,456 & 109386 & 494935 \\
\hline
\end{tabular}

\section{References}

1 .Nick Smith, Ph.D. Thesis, Speciation and Spectroscopy of the Uranyl and Tetravalent Plutonium Nitrate Systems: Fundamental Studies and Applications to Used Fuel Reprocessing, University of Nevada, Las Vegas, 2010

2 Jamie Warburton, Ph.D. Thesis, Spectroscopic Methods of Process Monitoring for Safeguards of Used Nuclear Fuel Separations, University of Nevada, Las Vegas, 2011.

3 Troy Robinson, Ph.D. Thesis, Equilibrium Speciation of Select Lanthanides in the Presence of Acidic Ligands in Homo- and Heterogeneous Solutions, University of Nevada, Las Vegas, 2011.

4 Warburton' J., Smith' N., and Czerwinski, K.: Method for Online Process Monitoring for Use in Solvent Extraction and Actinide Separations, Sep. Sci. Tech., 45(12-13), 1763-1768 (2010). 